\title{
Acyclic Retinoid Combined With Tenascin-C-derived Peptide Reduces the Malignant Phenotype of Neuroblastoma Cells Through N-Myc Degradation
}

\author{
KAZUKI OTSUKA ${ }^{1,2^{*}}$, MANABU SASADA $^{1,3^{*}}$, YU HIRANO $^{1}$, YUSUKE NOHARA $^{1}$, \\ TAKUYA IYODA ${ }^{4}$, YOSHIKAZU HIGAMI ${ }^{2,3}$, HIROAKI KODAMA ${ }^{5}$ and FUMIO FUKAI ${ }^{1,3}$ \\ ${ }^{1}$ Department of Molecular Patho-Physiology, Faculty of Pharmaceutical Sciences, \\ Tokyo University of Science, Noda, Japan; \\ ${ }^{2}$ Department of Molecular Pathology and Metabolic Disease, \\ Faculty of Pharmaceutical Sciences, Tokyo University of Science, Noda, Japan; \\ ${ }^{3}$ Translational Research Center, Research Institutes for Science and Technology, \\ Tokyo University of Science, Noda, Japan; \\ ${ }^{4}$ Department of Pharmacy, Faculty of Pharmaceutical Sciences, \\ Sanyo-Onoda City University, Sanyo-Onoda, Japan; \\ ${ }^{5}$ Department of Biochemistry, Faculty of Science and Engineering, Saga University, Saga, Japan
}

\begin{abstract}
Background/Aim: Despite intensive chemotherapy, the survival rates for high-risk neuroblastoma, most of which have MYCN amplification, remain low. Overexpression of $\mathrm{N}$-myc oncoprotein promotes expression of cancer-associated properties. We recently found that combination of all-trans retinoic acid (ATRA) with the $\beta 1$-integrin-activating peptide TNIIIA2 attenuated cancer-associated properties of neuroblastoma cells through N-Myc degradation. However, ATRA has serious side-effects and there are concerns about late adverse effects. The aim of this study was to examine the effects of the combination of acyclic retinoid (ACR) with TNIIIA2 on neuroblastoma. Materials and Methods: The effects of ACR and TNIIIA2 were examined by neuroblastoma cell proliferation and survival assays as well as by using a neuroblastoma xenograft model. The levels of N-Myc and cancer-associated malignant properties were assayed by western blot and colony formation assay, respectively. Results: Combining ACR, which is clinically safe, with TNIIIA2
\end{abstract}

\footnotetext{
*These Authors contributed equally to this study.

Correspondence to: Fumio Fukai, Ph.D., Department of Molecular Patho-Physiology, Faculty of Pharmaceutical Sciences, Tokyo University of Science, 2641 Yamazaki, Noda, Chiba 278-8510, Japan. Tel: +81 471241501 (Ext. 4645), Fax: +81 471213608, e-mail: fukai@rs.noda.tus.ac.jp
}

Key Words: Neuroblastoma, proto-oncogene, MYCN, N-Myc, retinoic acid, all-trans retinoic acid, acyclic retinoid, tenascin-C, integrin, ubiquitin-proteasome system. induced proteasomal degradation of $N-M y c$ and reduction of neuroblastoma cell malignant properties. An in vivo experiment showed therapeutic potential. Conclusion: ACRTNIIIA2 combination treatment may be efficacious and clinical safe chemotherapy for high-risk neuroblastoma.

Neuroblastoma is a common pediatric solid tumor; special attention has been given to the high-risk group of neuroblastoma patients with amplification of the $M Y C N$ proto-oncogene. Despite intensive chemotherapy, 5-year event-free survival rate remains below $30 \%$ (1). Excessive expression of N-Myc not only causes an aberrant neuronal differentiation during development, which is a central aspect of neuroblastoma genesis (2), but also contributes to the acquisition of malignant properties in neuroblastoma, including multidrug-resistance $(3,4)$. Therefore, N-Myc oncoprotein is considered to be a pivotal target molecule for high-risk neuroblastoma chemotherapy, while there is no effective drug targeting this oncoprotein (5). Besides the low therapeutic efficacy, current chemotherapy regimens for high-risk neuroblastoma have an additional clinical problem, namely that treatment of pediatric cancers including neuroblastoma using general anticancer drugs carries the risk of long-lasting side effects, so-called "late effects," which can occur many years after the completion of chemotherapy. Many cancer survivors, particularly infant patients, have various serious late effects (6). Differentiation therapy using all-trans retinoic acid (ATRA) is accepted as chemotherapy and has a low risk of late effects $(7,8)$; however, it does not confer sufficient therapeutic effects in high-risk MYCN- 
A

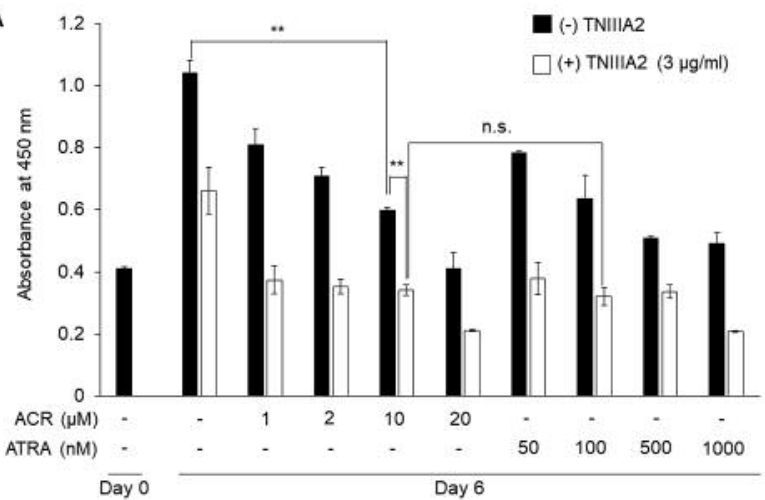

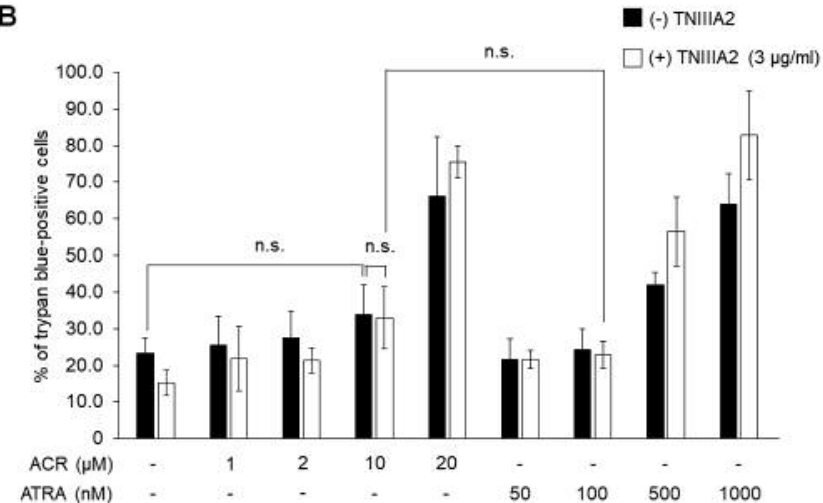

Figure 1. Effects of ACR, ATRA, and TNIIIA2 on cell survival/proliferation. (A and B) IMR-32 cells were cultured as indicated for 6 days. (A) The number of viable cells was evaluated by the WST assay as described in the Materials and Methods. (B) Cell viablity was evaluated by Trypan blue exclusion test and the percentages of cells positive to Trypan blue to total cells are shown: data are shown as the means $\pm S D(n=3)$. Statistical analysis was performed by non-parametric ANOVA and post hoc testing. ${ }^{*} p<0.01$; n.s.: Not significant.

amplified neuroblastoma. Achievement of N-Myc targeting would contribute to the establishment of mild chemotherapy for high-risk neuroblastoma with a low risk of late effects. We recently achieved molecular targeting of $\mathrm{N}$-Myc oncoprotein (9). Briefly, we combined the peptide TNIIIA2, which is capable of activating $\beta 1$-integrin (10), with ATRA, thereby inducing a marked decrease in N-Myc oncoprotein levels in neuroblastoma cells. Notably, this decrease in $\mathrm{N}$ Myc oncoprotein was accompanied by neuronal differentiation and reduced malignant properties of neuroblastoma cells (9). This finding may provide a new insight into future chemotherapy for high-risk neuroblastoma with $M Y C N$-amplification. However, a number of clinical reports have also indicated several severe side effects of ATRA, such as hyperleukocytosis, intracranial hypertension, and retinoic acid syndrome (11-13). Finding a minimally toxic compound that enables the targeting of N-Myc via combination with TNIIIA2, would contribute to the establishment of a mild chemotherapy for pediatric cancers including neuroblastoma.

Acyclic retinoid (ACR), a synthetic analog of retinoid (14, 15), which was previously developed for the chemoprevention of hepatocellular carcinoma (HCC) (1618), has an extremely low risk of side effects compared with ATRA $(16,18)$. We demonstrated that combining TNIIIA2 with ACR instead of ATRA can induce proteasomal degradation of $\mathrm{N}-\mathrm{Myc}$ oncoprotein in MYCN-amplified neuroblastoma cells and that the $\mathrm{N}$-Myc degradation is accompanied by a reduction in malignant properties. Furthermore, an in vivo experiment using a neuroblastoma xenograft model showed the therapeutic potential of this ACR-TNIIIA2 combination for neuroblastoma with no significant changes in body weight and tissue damage.

\section{Materials and Methods}

Cells. The human neuroblastoma cell line IMR-32 was obtained and incubated as described in our previous report (9).

Reagents. The synthetic TNIIIA2 peptide (RSTDLPGLKAATH YTITIRGVC), ATRA and MG-132 (Carbobenzoxy-L-leucyl-Lleucyl-L-leucinal) were purchased as described in our previous report (9). ACR (Peretinoin ${ }^{\circledR}$ ) was provided from Kowa Company, Ltd. (Nagoya, Japan).

Cell proliferation and survival. IMR-32 cells were cultured with minimum essential medium (MEM) (Thermo Fisher Scientific, Waltham, MA, USA) containing $1 \%$ fetal bovine serum (FBS) (SAFC Biosciences, Lenexa, Kansas, MO, USA) and ATRA or ACR in the presence or absence of TNIIIA2 in the plates coated with $2 \mu \mathrm{g} / \mathrm{ml}$ of fibronectin (Sigma, St. Louis, MO, USA). After incubation for 6 days, the viable cell number was evaluated by either WST assay using Cell counting kit-8 (Dojindo, Kumamoto, Japan) or conventional trypan blue exclusion test.

Western blotting. Western blotting was performed as described recently (9) using anti-N-Myc antibody (Merck Millipore, Burlington, MA, USA), anti-actin antibody (Sigma) and anti-Aurora A antibody (Cell Signaling Technology, Danvers, MA, USA).

Colony formation assay. Cancer-associated malignant property was evaluated by colony formation assay using soft agarose as previously described (9).

Neuroblastoma xenograft model. The animal procedure was approved by the Institutional Animal Care and Use Committee of Tokyo University of Science. IMR-32 cell suspension $\left(2 \times 10^{6}\right.$ cells $)$ containing EHS-gel (FUJIFILM Wako, Osaka, Japan) was injected subcutaneously in the left flank of each Balb-c nude mouse (Sankyo Labo Service Corporation, Tokyo, Japan). After the average tumor volume of all mice reached up to about $70 \mathrm{~mm}^{3}$, these mice were 
A

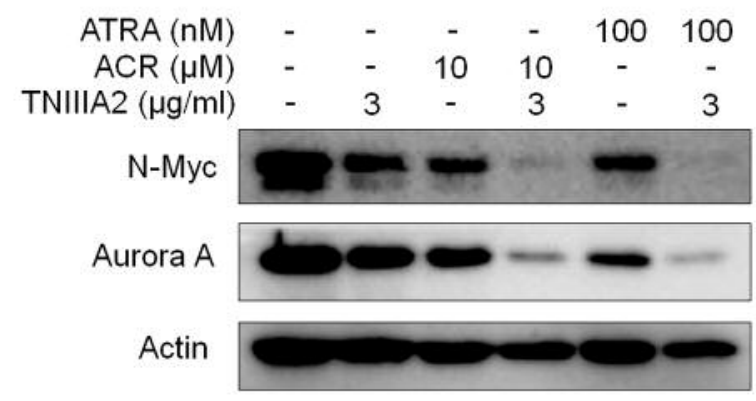

B

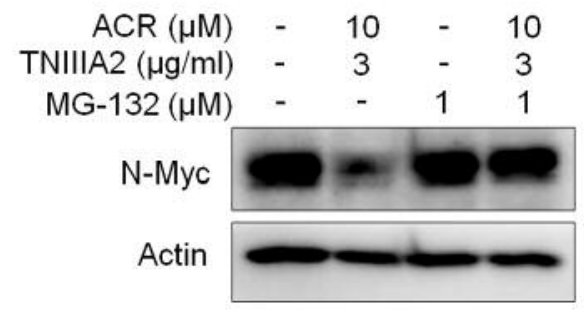

Figure 2. Proteasomal degradation of N-Myc protein induced by a combination of ACR and TNIIIA2. IMR-32 cells were cultured as indicated for 6 days before western blotting analysis. (A) Detection of N-Myc and Aurora A. (B) Detection of N-Myc. At day 5, MG-132 was added to the cultured media.

divided randomly to 3 groups (control, ACR and ACR-TNIIIA2). Each group was subjected to daily chemotherapy with vehicle, ACR or the ACR-TNIIIA2 combination initially for 1 week (day 0-6). Control group: Mice $(\mathrm{n}=3)$ were administrated with soybean oil (p.o.) as the vehicle of ACR and saline (alternately i.p. or i.v.) as the vehicle of TNIIIA2. ACR group: Mice $(n=3)$ were administrated with saline (alternately i.p. or i.v.) and ACR ( $2 \mathrm{mg}$ p.o./head). ACRTNIIIA2 group: Mice $(\mathrm{n}=3)$ were administrated with ACR $(2 \mathrm{mg}$ p.o./head) and TNIIIA2 (alternately $500 \mu \mathrm{g}$ i.p./head or $250 \mu \mathrm{g}$ i.v./head). Every mouse was observed every 3 days to calculate tumor volume and the body weight was measured at days $0,7,14$, 21 and 24 . The volume of tumor tissues was estimated by measuring tumor sizes and relative tumor volume was calculated, as described recently (9): volume $=\left(a^{2} \times b\right) / 2$, a: minor axis and $b$ : major axis, relative tumor volume $=$ the tumor volume at each day/the tumor volume at day 0 . Tumor tissues were collected at day 24 for western blotting analysis.

\section{Results and Discussion}

Proteasomal degradation of $N$-Myc protein by combining ACR with TNIIIA2. In our previous study, combination treatment of ATRA with TNIIIA2 induced proteasomal degradation of N-Myc protein in human neuroblastoma IMR32 cells with MYCN amplification (9). Prior to investigating whether ATRA can be substituted with ACR for its ability to induce degradation of N-Myc protein, we first compared the effects of ACR or ATRA in combination with TNIIIA2 on the survival and proliferation of IMR-32 cells to determine the optimal concentration of ACR without cytotoxic effects. As shown in Figure 1A, the proliferation of IMR-32 cells was suppressed by TNIIIA2 in combination with ACR in a concentration-dependent manner, to a similar extent as that of ATRA but both reached plateaus at concentrations of 1-10 $\mu \mathrm{M}$ ACR and 50-500 nM ATRA. Under these conditions, it was shown that cell death (as evaluated by a Trypan blue exclusion test) was dependent on ACR concentration, similar to that induced by ATRA; however, about $70 \%$ of IMR-32

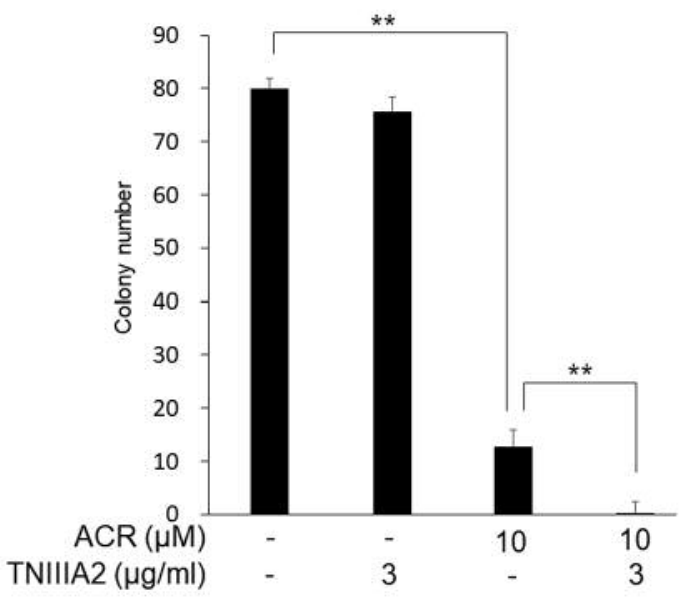

Figure 3. Suppression of anchorage-independent growth by the ACRTNIIIA2 combination. IMR-32 cells were cultured as indicated for 6 days and a soft agar formation assay was performed as described in the Materials and Methods. The results show the total amount of counts in 5 fields of each well at 4 weeks; data are shown as means $\pm S D(n=3)$. Statistical analysis was performed by non-parametric ANOVA and post hoc testing. $* * p<0.01$.

cells survived at ACR concentrations of $<10 \mu \mathrm{M}$ and ATRA concentrations of $<100 \mathrm{nM}$ (Figure 1B). Based on these results, we used ACR at $10 \mu \mathrm{M}$ and ATRA at $100 \mathrm{nM}$ in combination with TNIIIA2 $(3 \mu \mathrm{g} / \mathrm{ml})$, in our experiments.

We next investigated the combined effect of ACR and TNIIIA2 treatment on the intracellular N-Myc expression levels. IMR-32 cells were treated with TNIIIA2 in the presence or absence of ATRA or ACR for 6 days. As reported recently (9), treatment with TNIIIA2 or ATRA alone slightly decreased the intracellular levels of N-Myc protein, while the combination of the two caused a remarkable decrease (Figure 2A). Similarly, ACR also slightly reduced 
A

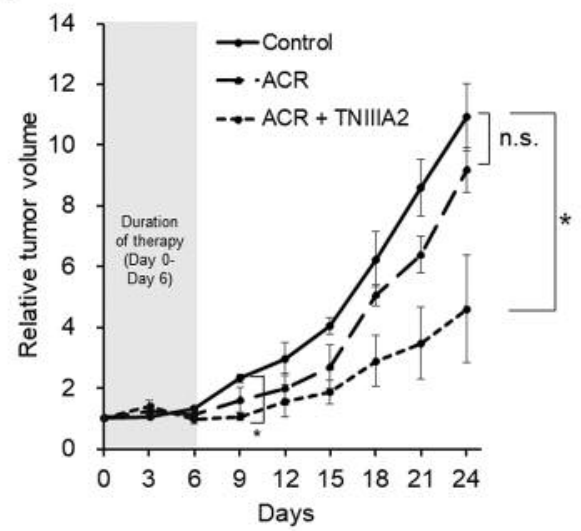

B

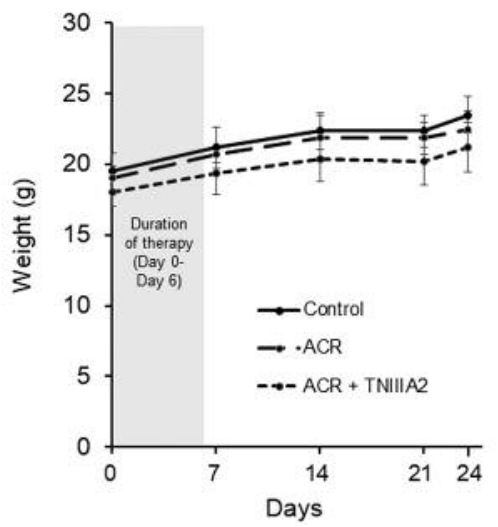

C

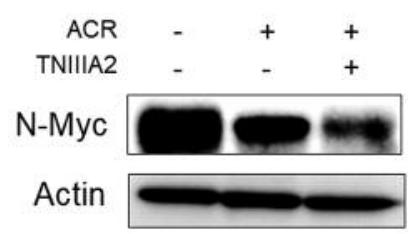

Figure 4. Combined administration of ACR and TNIIIA2 suppressed tumor growth in xenograft model mice. (A-C) Preparations of neuroblastoma xenograft model and chemotherapy with vehicle, ACR or the ACR-TNIIIA2 combination were performed as described in the Materials and Methods. (A) Relative tumor volume of each group in chemotherapy; data are shown as the means $\pm S D(n=3)$. Statistical analysis was performed by MannWhitney U-test. ${ }^{*} p<0.05$; n.s.: Not significant. (B) Monitoring of body weight. (C) Western blotting analysis of N-Myc in tumor tissues on day 24.

$\mathrm{N}-\mathrm{Myc}$ protein levels, but the N-Myc protein levels were significantly decreased when combined with TNIIIA2. As shown in Figure 2B, the decreased expression of N-Myc protein caused by the combination of ACR and TNIIIA2 was completely reversed by the addition of MG-132, a proteasomal inhibitor, suggesting that the proteasomal degradation of N-Myc protein was at least attributable to the decreased levels of N-Myc protein. In the process of proteasomal degradation of N-Myc, Aurora A prevents degradation by directly binding and stabilizing ubiquitinated N-Myc (19). Treatment of IMR-32 cells with TNIIIA2 or ACR alone showed no significant effects on the intracellular expression levels of Aurora A protein, while Aurora A protein levels were remarkably decreased by the combination of the two, in parallel with the decrease in N-Myc protein (Figure 2A). These results suggest that combining ACR with TNIIIA2 enables molecular targeting of N-Myc protein based on proteasomal degradation.

Therapeutic potential of molecular targeting of N-Myc by the ACR-TNIIIA2 co-treatment in MYCN-amplified neuroblastoma. Since highly expressed N-Myc protein is associated with the acquisition of oncogenic properties in neuroblastoma cells (2), it is expected that the ACR-TNIIIA2 combination treatment may influence the cancer-associated malignant properties of IMR-32 cells. We therefore evaluated the effect of the ACRTNIIIA2 combination on anchorage-independent cell proliferation, which is one of the malignant properties of cancer cells (20). IMR-32 cells were pre-treated with ACR in the presence or absence of TNIIIA2 for 6 days and then a soft agar colony formation assay was performed. Pre-treatment with TNIIIA2 showed no significant effect on colony formation, while ACR remarkably reduced the number of colonies (Figure 3). Furthermore, the ability to form colonies nearly disappeared when ACR was combined with TNIIIA2 (Figure 3).

Based on these in vitro results, the therapeutic effect of the ACR-TNIIIA2 combination was examined using a neuroblastoma xenograft mouse model. IMR-32 cells were transplanted subcutaneously into the left flanks of Balb-c nude mice. Then, tumor-bearing mice were randomly divided into three groups: control, ACR and ACR-TNIIIA2 combination. Drugs were administered every day for one week, and tumor volumes were monitored every 3 days for 24 days. While no significant difference in tumor growth was observed following ACR administration compared with the control, the ACR-TNIIIA2 combination clearly suppressed tumor growth (Figure 4A), without significant changes in body weight (Figure 4B) or noticeable tissue changes in the mice. This suppression of tumor growth was accompanied by a remarkable decrease in N-Myc protein levels in the tumor tissues of the mice at day 24 (Figure 4C). Thus, the beneficial effects of targeting N-Myc protein by the ACRTNIIIA2 combination were verified both in vitro and in vivo.

This study demonstrated that proteasomal degradation of $\mathrm{N}-\mathrm{Myc}$ can be induced by ACR, instead of ATRA, in combination with TNIIIA 2 (Figure 2A). ACR, which is considered to have a much lower risk of side effects than ATRA $(16,18)$, was developed as a drug to prevent recurrence of HCC (16-18). Recently, Qin et al. identified MYCN as a target molecule of ACR for the prevention of HCC (21). They found that ACR reduces the expression of MYCN at both the protein and gene level, resulting in specific suppression of the proliferation of HCC cells with high MYCN expression, although the molecular mechanism 
was not clarified. In addition, MYCN is also amplified in cancers other than neuroblastoma, such as medulloblastoma and small-cell lung cancer, and correlates with poor prognosis (22). Taken together with our results, treatment combining TNIIIA2 and ACR could achieve more efficient prevention of recurrent $\mathrm{HCC}$ and improve poor clinical profiles in MYCN-amplified cancers via molecular targeting of N-Myc.

Our series of studies, including our most recent report (9), suggest that combining retinoic acid or its derivatives with TNIIIA2 may provide a novel therapeutic strategy for highrisk MYCN-amplified neuroblastoma based on N-Myc degradation. Using ACR as a functional retinoid instead of ATRA may offer an effective cancer chemotherapy with a low risk of side effects.

\section{Conflicts of Interest}

The Authors declare no conflicts of interest regarding this study.

\section{Authors' Contributions}

K.O. and M.S. designed and performed experiments, analyzed the data and wrote the manuscript. Y.H. and Y.N. performed experiments. T.I., Y.H., H.K. and F.F. organized the study, and reviewed and edited the manuscript.

\section{Acknowledgements}

The Authors acknowledge the support of the Japan Science and Technology Agency (Adaptable and Seamless Technology Transfer Program through Target-driven R\&D) and thank Dr. Akimune Asanuma, Dr. Masanao Watanabe, Dr. Shingo Yanagida and Naoto Ishibashi of KOWA COMPANY, LTD. for their kind help in this study.

\section{References}

1 Cohn SL, Pearson ADJ, London WB, Monclair T, Ambros PF, Brodeur GM, Faldum A, Hero B, Iehara T, Machin D, Mosseri V, Simon T, Garaventa A, Castel V and Matthay KK: The international neuroblastoma risk group (INRG) classification system: an INRG Task Force report. J Clin Oncol 27(2): 289297, 2009. PMID: 19047291. DOI: 10.1200/JCO.2008.16.6785

2 Nakagawara A, Li YY, Izumi H, Muramori K, Inada H and Nishi M: Neuroblastoma. Jpn J Clin Oncol 48(3): 214-241, 2018. PMID: 29378002. DOI: 10.1093/jjco/hyx176

3 Chesler L, Schlieve C, Goldenberg DD, Kenney A, Kim G, McMillan A, Matthay KK, Rowitch D and Weiss WA: Inhibition of phosphatidylinositol 3-kinase destabilizes mycn protein and blocks malignant progression in neuroblastoma. Cancer Res 66(16): 8139-8146, 2006. PMID: 16912192. DOI: 10.1158/ 0008-5472.CAN-05-2769

4 Norris MD, Bordow SB, Haber PS, Marshall GM, Kavallaris M, Madafiglio J, Cohn SL, Salwen H, Schmidt ML, Hipfner DR, Cole SPC, Deeley RG and Haber M: Evidence that the mycn oncogene regulates mrp gene expression in neuroblastoma. Eur J Cancer 33(12): 1911-1916, 1997. PMID: 9516823. DOI: 10.1016/S0959-8049(97)00284-0
5 Chen H, Liu HD and Qing GL: Targeting oncogenic myc as a strategy for cancer treatment. Signal Transduct Target Ther 3: 5, 2018. PMID: 29527331. DOI: 10.1038/s41392-0180008-7

6 Friedman DN and Henderson TO: Late effects and survivorship issues in patients with neuroblastoma. Children 5(8): 107, 2018. PMID: 30082653. DOI: 10.3390/children5080107

7 Adamson PC: Clinical and pharmacokinetic studies of all-transretinoic acid in pediatric-patients with cancer. Leukemia $8(11)$ : 1813-1816, 1994. PMID: 7967727.

8 Adamson PC, Matthay KK, O'Brien M, Reaman GH, Sato JK and Balis FM: A phase 2 trial of all-trans-retinoic acid in combination with interferon-alpha $2 \mathrm{~A}$ in children with recurrent neuroblastoma or Wilms tumor: A pediatric oncology branch, NCI and children's oncology group study. Pediatr Blood Cancer 49(5): 661-665, 2007. PMID: 16900483. DOI: 10.1002/ pbc. 21011

9 Otsuka K, Sasada M, Iyoda T, Nohara Y, Sakai S, Asayama T, Suenaga Y, Yokoi S, Higami Y, Kodama H and Fukai F: TNIIIA2 with all-trans retinoic acid accelerates N-Myc protein degradation and neuronal differentiation in MYCN-amplified neuroblastoma cells. Am J Cancer Res 9(2): 434-448, 2019. PMID: 30906641.

10 Saito Y, Imazeki H, Miura S, Yoshimura T, Okutsu H, Harada Y, Ohwaki T, Nagao O, Kamiya S, Hayashi R, Kodama H, Handa $\mathrm{H}$, Yoshida $\mathrm{T}$ and Fukai F: A peptide derived from tenascin-C induces $\beta 1$ integrin activation through syndecan-4. J Biol Chem 282(48): 34929-34937, 2007. PMID: 17901052. DOI: 10.1074/ jbc.M705608200

11 Warrell RP, Frankel SR, Miller WH, Scheinberg DA, Itri LM, Hittelman WN, Vyas R, Andreeff M, Tafuri A, Jakubowski A, Gabrilove J, Gordon MS and Dmitrovsky E: Differentiation therapy of acute promyelocytic leukemia with tretinoin (alltrans-retinoic acid). N Engl J Med 324(20): 1385-1393, 1991. PMID: 1850498. DOI: 10.1056/NEJM199105163242002

12 Labrador J, Puig N, Ortin A, Gutierrez NC and Gonzalez-Diaz M: Multiple cranial neuropathy and intracranial hypertension associated with all-trans retinoic acid treatment in a young adult patient with acute promyelocytic leukemia. Int J Hematol 96(3): 383-385, 2012. PMID: 22767142. DOI: 10.1007/s12185-0121134-6

13 Frankel SR, Eardley A, Heller G, Berman E, Miller WH, Dmitrovsky E and Warrell RP: All-trans-retinoic acid for acute promyelocytic leukemia - Results of the New York Study. Ann Intern Med 120(4): 278-286, 1994. PMID: 8291820. DOI: 10.7326/0003-4819-120-4-199402150-00004

14 Muto Y, Moriwaki H and Omori M: In vitro binding affinity of novel synthetic polyprenoids (polyprenoic acids) to cellular retinoid-binding proteins. Gan 72(6): 974-977, 1981. PMID: 7200437.

15 Araki H, Shidoji Y, Yamada Y, Moriwaki H and Muto Y: Retinoid agonist activities of synthetic geranyl geranoic acidderivatives. Biochem Biophys Res Commun 209(1): 66-72, 1995. PMID: 7726866. DOI: 10.1006/bbrc.1995.1471

16 Muto Y, Moriwaki H, Ninomiya M, Adachi S, Saito A, Takasaki KT, Tanaka T, Tsurumi K, Okuno M, Tomita E, Nakamura T and Kojima T: Prevention of second primary tumors by an acyclic retinoid, polyprenoic acid, in patients with hepatocellular carcinoma. N Engl J Med 334(24): 1561-1567, 1996. PMID: 8628336. DOI: $10.1056 /$ NEJM199606133342402 
17 Takai K, Okuno M, Yasuda I, Nishiwaki RM, Uematsu T, Tsurumi H, Shiratori Y, Muto Y and Moriwaki H: Prevention of second primary tumors by an acyclic retinoid in patients with hepatocellular carcinoma - updated analysis of the long-term follow-up data. Intervirology 48(1): 39-45, 2005. PMID: 15785088. DOI: $10.1159 / 000082093$

18 Okita K, Izumi N, Matsui O, Tanaka K, Kaneko S, Moriwaki H, Ikeda K, Osaki Y, Numata K, Nakachi K, Kokudo N, Imanaka K, Nishiguchi S, Okusaka T, Nishigaki Y, Shiomi S, Kudo M, Ido K, Karino Y, Hayashi N, Ohashi Y, Makuuchi M, Kumada $\mathrm{H}$ and Peretinoin Study Group: Peretinoin after curative therapy of hepatitis C-related hepatocellular carcinoma: a randomized double-blind placebo-controlled study. J Gastroenterol 50(2): 191-202, 2015. PMID: 24728665. DOI: 10.1007/s00535-0140956-9

19 Otto T, Horn S, Brockmann M, Eilers U, Schuttrumpf L, Popov N, Kenney AM, Schulte JH, Beijersbergen R, Christiansen H, Berwanger B and Eilers M: Stabilization of N-Myc is a critical function of Aurora A in human neuroblastoma. Cancer Cell 15(1): 67-78, 2009. PMID: 19111882. DOI: 10.1016/j.ccr.2008. 12.005
20 Mori S, Chang JT Andrechek ER, Matsumura N, Baba T, Kim JW, Gatza M, Murphy S and Nevins JR: Anchorage-independent cell growth signature indentifies tumors with metastatic potential. Oncogene 28(31): 2796-2805, 2009. PMID: 19483725. DOI: $10.1038 /$ onc 2009.139

21 Qin XY, Suzuki H, Honda M, Okada H, Kaneko S, Inoue I, Ebisui E, Hashimoto K, Carninci P, Kanki K, Tatsukawa H, Ishibashi N, Masaki T, Matsuura T, Kagechika H, Toriguchi K, Hatano E, Shirakami Y, Shiota G, Shimizu M, Moriwaki H and Kojima S: Prevention of hepatocellular carcinoma by targeting mycn-positive liver cancer stem cells with acyclic retinoid. Proc Natl Acad Sci USA 115(19): 4969-4974, 2018. PMID: 29686061. DOI: $10.1073 /$ pnas.1802279115

22 Beltran H: The n-myc oncogene: Maximizing its targets, regulation, and therapeutic potential. Mol Cancer Res 12(6): 815-822, 2014. PMID: 24589438. DOI: 10.1158/1541-7786.MCR-13-0536

Received May 6, 2019

Revised June 1, 2019

Accepted June 3, 2019 